\title{
The Relationship Between Bitcoin and Stock Market
}

\author{
Xin Wang, Saint Peter's University, USA \\ Xi Chen, Beijing University of Civil Engineering and Architecture, China \\ Peng Zhao, Intelligentrabbit, LLC., USA
}

\begin{abstract}
This article analyzes the relationship between Bitcoin and the stock market by using a vector autoregressive model. To enhance the impulse response signal, the Sliding Window technique is applied. Study results show the relationship between Bitcoin and the stock market. First, the S\&P 500 has a relatively significant effect on Bitcoin, while the influence caused by the S\&P 500 is weak. In addition, after involving the Sliding Window technique, the effects caused by the standard deviation of the S\&P 500 and the mean of the Dow Jones are remarkably strong on the mean of Bitcoin and the standard deviation of the S\&P 500 has a comparatively significant effect on the standard deviation of Bitcoin as well. Generally, the S\&P 500 and the Dow Jones indexes have an advantageous effect on Bitcoin. Financial investment can be made based on this model and conclusion.
\end{abstract}

\section{KEYWORDS}

Bitcoin, Price Return, Sliding Window Technique, Stock Market, VAR Model

\section{INTRODUCTION}

Bitcoin, regarded as a new type of new digital currency that could be used on transaction among different parties, has attracted increasing attention from scholars and financial experts. Satoshi Nakamoto (2008) first invented Bitcoin, the appearance of which realized decentralized transaction. Bitcoin allows online payment to be transacted directly from one party to another without getting through an intermediate financial institution, while traditional finance resists the trust-in-third-party mechanism centralized on the financial institution. Even though the public believe Bitcoin finance has the potential to replace the antiquated finance paradigm, Bitcoin and traditional finance are currently coexisting. In Bitcoin finance, more reliable trust is established not by authority intermediaries, but by network consensus, cryptography, digital signature, which reduces high transaction costs. However, the possibility of system failures and attacks or fraud behavior is always unavoidable in online transactions. In this case, traditional finance stands in a more advantageous position.

Gandal et al. (2018) provided analyses for the Bitcoin Price in recent years and argued that Bitcoin experienced both rising and falling tendency in the past years. Price of Bitcoin gets a falling shock, following large investments in Bitcoin. The volatility of Bitcoin has graphical similarity with that of the stock market. Many related predictive analysis methods, proposed by economists to predict the stock market, have also been used to forecast Bitcoin price volatility. With the development of the Bitcoin economy and more and more attentions from the industry of financial investment, whether the relationship between Bitcoin and the stock market has become a valuable research subject, although

This article, originally published under IGI Global's copyright on April 1, 2020 will proceed with publication as an Open Access article starting on February 1, 2021 in the gold Open Access journal, International Journal of Operations Research and Information Systems (converted to gold Open Access January 1, 2021), and will be distributed under the terms of the Creative Commons Attribution License (http:// creativecommons.org/licenses/by/4.0/) which permits unrestricted use, distribution, and production in any medium, provided the author of the original work and original publication source are properly credited. 
this subject has not been mentioned by experts and scholars widely. The relationship between the stock market and Bitcoin will help investors to make their investment strategy and find out possible influencing factors on the Bitcoin market.

The study of this paper has made significant contributions to the academic field and financial market analysis as follows:

- We present a groundbreaking study of the relationship between Bitcoin and the stock market over time based on Vector Autoregressive model of Time Series. The impulse response is involved as signal to observe the reaction of this dynamic system of Bitcoin and stock market in response to some change caused by relative parameters.

- Sliding Window technique is utilized to enhance impulse response signals. With the size changes of sliding window, the loudness of the impulse response signal varies as well.

- Big Data technology is applied in data processing. The study applied Yahoo API for batch data collection of three stock indexes.

In this paper, we firstly introduce the development background of Bitcoin as one type of cryptocurrency based on blockchain protocol, recall the existing economic modeling strategies, including Random Walk hypothesis, SVR, RF, ANN, etc., used for stock market forecasting and further explore the solutions of VAR, impulse response and sliding window to figure out non-stationary time series with the feature of seasonal volatility, corresponding to the data of stock market and Bitcoin, in Section 2. In Section 3, data collection of the three main stock indexes is performed through Yahoo Finance API on Python and then we study the time series tendency over time for each series and their respective price return series. We describe in detail about the basic modeling framework of VAR model on four variables in Section 4. In Section 5, the results of impulse response and variance decomposition are shown, and further efforts are made to obtain impulse response among variables through setting up sliding windows. Discussion for further study is elaborated in Section 6. Finally, we conclude a dynamic relationship between Bitcoin and the stock market.

\section{BACKGROUND}

As a kind of digital currency, Bitcoin was firstly invented and proposed by Satoshi Nakamoto in 2008 . He proposed a peer-to-peer network solution, which can handle transactions into a chain of hash-based proof-of-work. In the process, an unchangeable record will be formed even without proof-of-work. Bitcoin is based on the blockchain protocol to process transactions of the digital Bitcoin currency between two parties. Eyal et al. (2016) outlined the Bitcoin-NG scalable blockchain protocol and designed quantitative metrics that can be used to evaluate Nakamoto consensus protocols. BitcoinNG scalable blockchain protocol finally can guarantee higher output and lower latency through its scalability and robustness. Since the Bitcoin cryptocurrency records all transactions in the blockchain, which is a public distributed log, its security plays an important role in the whole transaction process. Eyal and Sirer (2018) pointed out that Bitcoin's mining protocol is not incentive-compatible and presented a strategy called Selfish Mining that can enable more revenue by a minority pool. A majority of Bitcoin researches focus on cryptography and security of the Bitcoin transaction, but rare of them involve the relationship between Bitcoin and the stock markets.

For a long time, the financial community and academia have generally believed that stock price and its volatility are forecastable. The most well-known and initial theories in stock prediction are Random Walk hypothesis (Malkiel \& Fama, 1970; Malkiel, 2003) and the Efficient Market hypothesis (Jensen, 1978), which points out that the existing information set would influence the current price of the stock. Therefore, stock prices couldn't be predicted accurately by historical price information. Consequently, Malkiel concluded that economic profits cannot gain as the result of stock prices 
randomness except considering the excessive sort of risk. The research based on the behavior of stockmarket prices (Fama et al., 1965) examined the Random Walk hypothesis and discussed its attributes: (a) consecutive price changes is with independence. (b) the price change is by a certain probability distribution. With the improvement of technology and academic ability, progressively more machine learning algorithms and models have been applied in the research and analysis of stock prices in recent years. Support Vector Regression (SVR) based on chaotic mapping firefly algorithm (Kazem et al. 2013) is proposed to forecast stock market price, which established unobserved phase space dynamics through a delay coordinate embedding method, and employed a chaotic firefly algorithm to get optimization for SVR hyperparameters to predict the stock market price. All these models were employed based on the precondition that the predicted time series are linear and stationary, which will, to a certain extent, cause the forecast result to deviate from the actual situation. Besides, the fusion of multiple machine learning techniques has been utilized to predict the stock market (Patel et al. 2015). A two-stage fusion hybrid model could be set up: the first stage fusion use SVR and the second stage fusion include Artificial Neural Network (ANN), Random Forest (RF) and SVR leading to SVR-ANN, SVR-RF and SVR-SVR fusion forecast models. This hybrid model has efficiently and successfully decreased the error in the performance of prediction. Furthermore, Hafezi, Shahrabi, and Hadavandi (2015) proposed a bat-neural network multi-agent system (BNNMAS) with four layers for stock price prediction. They applied a metaheuristic Bat algorithm to train the artificial neural network (ANN) and eventually got high accuracy of prediction indicated by MAPE statistic calculation. With the further application of deep learning, Zhou, Pan, Hu, Tang and Zhao (2018) stated stock forecasting model GAN-FD based on the deep learning algorithm, which attained remarkable prediction performance through optimizing training, reducing direction forecast loss and prediction error loss. Due to the non-linearity and dynamic state of stock market forecasting, Basak, Saha, Kar, Khaidem, and Dey (2019) built a predictive model based on Random Forest and XGB classifiers with high robustness, indicated by diverse standard parameters including accuracy, recall, specificity, and F-score. Their proposed model reasonably reduced the risk of stock market investment through analyzing the returns of stock and got higher accuracy of direction prediction of the stock market, comparing to any other applied algorithm models. However, in many studies on stock market forecasting, the impact or potential correlation between Bitcoin and the stock market has been hardly discussed.

Since stock market data can be regarded as non-stationary time series data as well, the time series model is an ideal method for studying the stock market. Time series models are often used to predict the future value of stocks based on previously observed values. Sadaei, Guimaraes, Silva, Lee, and Eslami (2017) provided a hybrid method based on exponential fuzzy time series to improve the accuracy of forecasts for the seasonal and nonlinear time series. To figure out any limitations, such as heavy-tails, long-range dependence, and temporal asymmetries, etc., Leonarduzzi, Rochette, Bouchaud, and Mallat (2019) proposed wavelet-based maximum scattering entropy model to deal with non-stationary time series and discussed how phase-harmonic correlations can keep track with the temporal asymmetries. In macroeconomic forecasting, the Vector Auto-Regression (VAR) model has been successfully used for the multivariate time series analysis. Deb (2019) further studied Degras, $\mathrm{Xu}$, Zhang and Wu (2011)'s theoretical research about a method to test the possibility of that two multivariate time series are from same VAR process model and developed a new time series clustering algorithm without involving any predetermined number of clusters to test methods. Moreover, the impulse response function is frequently used to analyze the response of various variables in the VAR model to different shock influences. Reusens and Croux (2017) studied whether the impulse response of linearly transformed variables is economically comparative. Since time series data typically has seasonal volatility, the sliding window algorithm is applied for short-term future predictions. The performances of three deep learning had been compared based on a sliding window approach for the forecasting of stock price fluctuation in Selvin, Vinayakumar, Gopalakrishnan, Menon, and Soman (2017)'s research. However, in these financial and algorithmic researches, the latent relationship between Bitcoin and the stock market has never been involved. 


\section{DATA SUMMARY}

This study obtains the data from two sources. Data of Bitcoin transaction flowing is available from Cryptocurrency Historical Prices on Kaggle. Cryptocurrency Historical Prices dataset covered the prices of Bitcoin, Ethereum, Ripple and Bitcoin cash, traced on each day from February 23, 2013 to February 20, 2018. Stock price. Stock Price Historical data is captured on daily transaction of three major stock market indices (S\&P 500, NASDAQ and Dow Jones Industrial Average) through Yahoo Finance API on Python, the time spans of which are respectively from March 4, 1957, February 8, 1971, and May 26, 1896 to February 20, 2018. To study the relationship over time between Bitcoin and Stock Price, these two data sources must be matched in the Bitcoin's shorter time interval and remove the data without the stock transaction. Figure 1 shows a time series plot of Bitcoin, S\&P 500, NASDAQ and Dow Jones Industrial Average with 2013 days from February 2010 to February 2018.

Since the data of Bitcoin, S\&P 500, NASDAQ, and Dow Jones is measured on different scales, which will affect the results of data analysis. Data Standardization is required to ensure the comparability among the four indicators. Based on the continuity of time series data, the Mean normalization method is suggested to normalize Bitcoin, S\&P 500, NASDAQ and Dow Jones data. The conversion function is as follows:

$$
X^{\prime}=\frac{X-\bar{X}}{\max (X)-\min (X)}
$$

After the original data is processed by data standardization, we can see in Figure 1 that each indicator is in the same order of magnitude, which is suitable for comprehensively comparative evaluation.

Figure 1 shows Bitcoin market price started to continuously increase from 2017, peaked on December 18, 2017, and sharply declined until 2018. Similar plunge from 2017 to 2018 has occurred in the graphs of S\&P 500, NASDAQ and Dow Jones as well, which have reflected the potential correspondence between Bitcoin market and the stock market. High volatility has been illustrated in the plot of S\&P 500, NASDAQ and Dow Jones, which means stock price fluctuate dramatically. Therefore, aiming to further analysis and observation of time series of Bitcoin and stock price, we calculate the price return by utilizing the following logarithmic identity:

$$
\text { Logreturn }=\log \left(\frac{P_{t 1}-P_{t 0}}{P_{t 0}}\right)=\log P_{t 1}-\log P_{t 0}
$$

In Figure 2, time series of price return based on Bitcoin, S\&P 500, NASDAQ and Dow Jones indicate each of the series has the zero mean with non-tendency of high stationary on time series.

\section{METHODOLOGY}

In this study, the main modeling foundation is based on the Vector Autoregressive Model (VAR). Four variables are involved in this VAR model, including BT (Bitcoin), SP (S\&P 500), ND (NASDAQ) and DJ (Dow Jones). Define a four-dimensional column vector as follows:

$$
y_{m}=(B T, S P, N D, D J)^{\prime}
$$


Figure 1. Time series plot. Bitcoin fell sharply from late 2017 to early 2018, while S\&P 500, NASDAQ and Dow Jones indexes continued to rise.

\section{Time Series Plot for Bicoin, S\&P 500, NASDAQ, and Dow Jones}
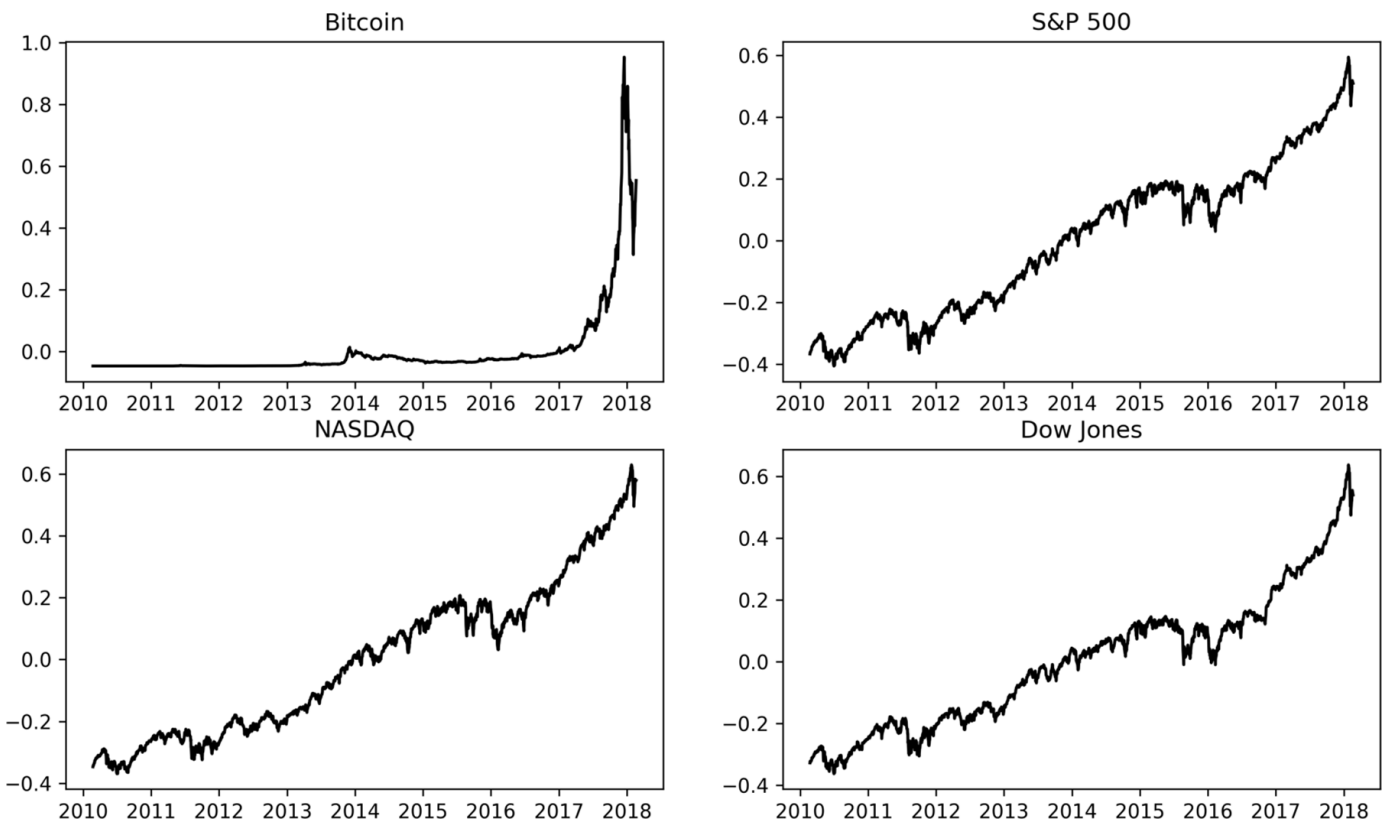

The VAR model can be expressed as below:

$y_{m}=B_{1} y_{m-1}+\ldots+B_{k} y_{m-k}+C x_{m}+\mathcal{E}_{m}, m=1,2, \ldots, M$

k is lag order. $B_{1}, \ldots, B_{k}$, and $C$ are parameter matrices. $M$ is the data size. $\mathcal{E}_{m}$ represents vector of errors.

The features of impulse response functions and variance decomposition allow tracing the evolution of endogenous variables corresponding to a shock in one or more variables. With the equation of evolution, the logic is organized as the following process. Let $R$ as a lag parameter.

$$
R y_{m}=y_{m-1}
$$

With Equation (4), the VAR model can be evolved as below:

$$
\left(P_{t}-B_{1} R-\ldots-B_{k} R^{k}\right) y_{m}=\mathcal{E}_{m}
$$

Invert this transformed VAR model formula:

$$
y_{m}=\left(P_{t}-B_{1} R-\ldots-B_{k} L^{k}\right)^{-1} \mathcal{E}_{m}=\left(P_{t}+D_{1} R+D_{2} R^{2}+\ldots\right) \mathcal{E}_{m}
$$


Figure 2. Price return plot. Compared to time series plot, price return shows relative stationery.

Price Return for Bicoin, S\&P 500, NASDAQ, and Dow Jones
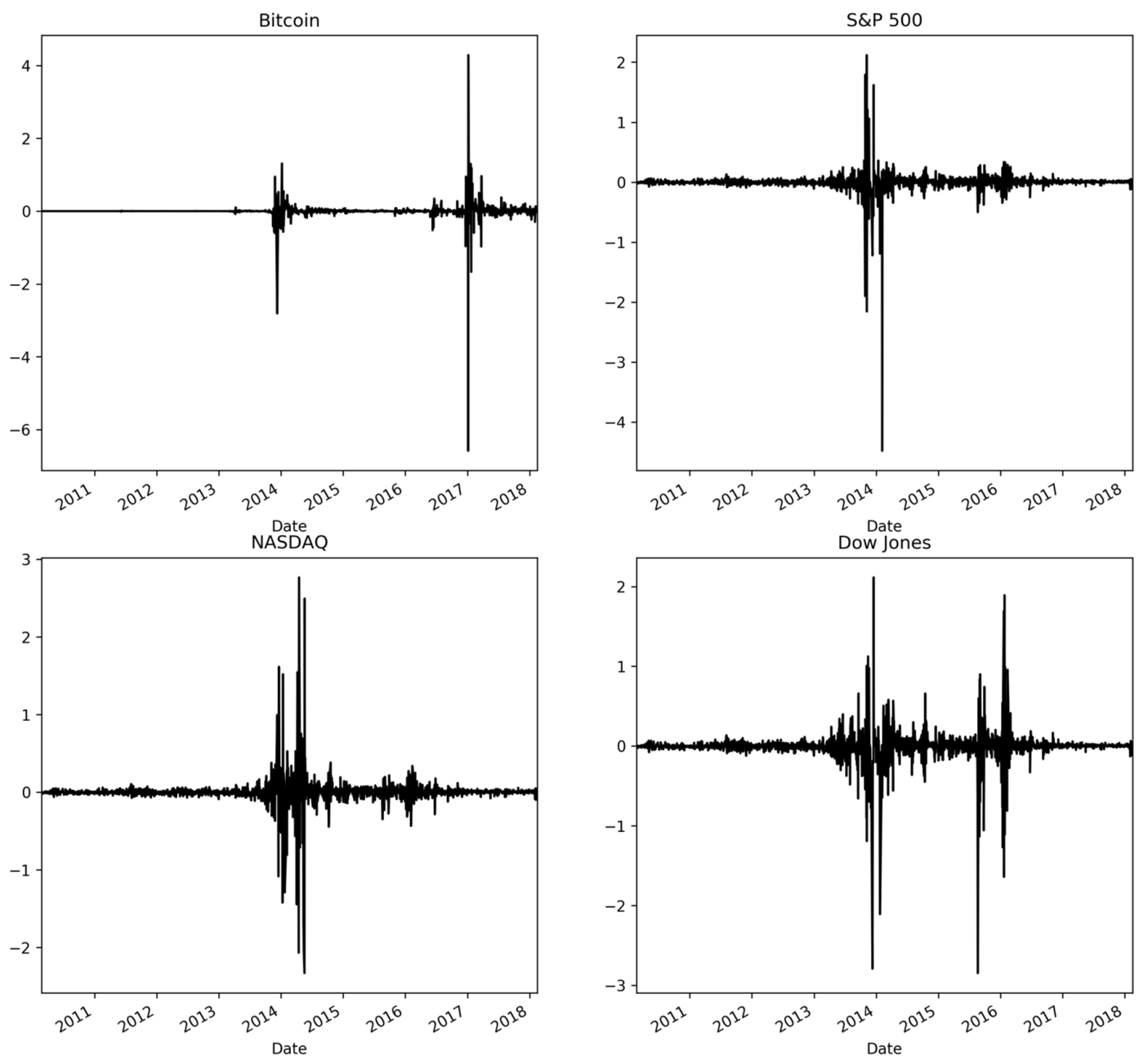

Each variable can be written as:

$y_{n_{t h} m}=\sum_{i=1}^{4}\left(D_{n_{t h} i}^{0} \mathcal{E}_{i m}+D_{n_{t h} i}^{1} \mathcal{E}_{i m-1}+\ldots\right), m=1,2, \ldots, M$

where $D_{n_{t h} i}^{l}$ is the $n_{t h}$ row and $i_{t h}$ line element of $D_{l}$, which can be symbolized as:

$$
D_{n_{t h} i}^{l}=\frac{\partial C E_{m+l}}{\partial \mathcal{E}_{i m}}
$$


The above partial derivatives show the response of $C E_{m+l}$ to a one-time impulse in $y_{i m}$ with all other variables held constant. The response of $n_{\text {th }}$ variable to a unit shock on variable $i$ could be graphically depicted the dynamic interrelationships with the system, the process of which is called Impulse Response Function (IRF).

Variance decomposition shows the extent that a shock to one variable impacts the variance. It determines the contribution of varieties of shocks to other variables in the autoregression. Its essential logic is as follows: based on the total $\mathcal{E}_{i m}$ 's effect on $C E_{m}$ from (7), the variances of $C E_{m}$ without correlations among $\mathcal{E}_{i}$ are

$$
E\left[\left(D_{n_{t h} \mathcal{E}_{i m}}^{0} \mathcal{E}_{i m}+D_{n_{t h} i}^{1} \mathcal{E}_{i m-1}+\ldots\right)^{2}\right]=\sum_{l=0}^{\infty}\left(D_{n_{t h} i}^{l}\right)^{2} \sigma_{i i}, i=1,2,3,4
$$

Moreover, the variance of $\partial C E_{m+l}$ under the condition of no correlations is:

$$
\operatorname{Var}\left(\partial C E_{m+l}\right)=\sum_{i=1}^{4} \sum_{l=0}^{\infty}\left(D_{n_{t h i} i}^{l}\right)^{2} \sigma_{i i}, m=1,2, \ldots, M
$$

\section{EXPERIMENTAL DESIGN AND ANALYSIS}

\section{Application of VAR Model}

As a method to study the influencing indicators, VAR model are widely applied in various fields. Adenomon and Oyejola (2013) studied the impact of agriculture and industrialization on GDP using VAR and SVAR models, which experimentally concluded the significant role performed by agriculture and industry sector to GDP growth and a greater effect from agriculture was shown through forecast error variance decompositions. In psychology field, Bringmann et al. (2018) used the TV-VAR model to explore emotion dynamics and their changes over time.

In this study, with four variables involving BT, SP, ND, and DJ, a VAR model is built to analyze the dynamic relationships. Lag order is selected at 18 and 3, according to AIC and BIC respectively. VAR (3) can be chosen as the more optimal model based on BID of lag order selection parameters since BIC approaches to the true model among the set of results.

The impulse response results are plotted in Figure 3. The shock effect to Bitcoin by Bitcoin itself demonstrates that it has an originally strong positive impact but a negative effect at lag 1 . The effect shock gradually augments from lag 2 to lag 4 and then flattens out. None of S\&P 500, NASDAQ and Dow Jones affects the beginning. The impact from S\&P 500 to Bitcoin has a negative effect at lag 1 and then a slightly positive effect at lag 4, while the impact from Dow Jones changes in the opposite trend. Dow Jones has a remarkably positive effect at lag 1 and a negative effect at lag 4 . NASDAQ has no significant effect to Bitcoin until lag 4, at which the impact is positive. In general, the impact effect from S\&P 500 or Dow Jones is greater than that of NASDAQ. Moreover, more attentions should be paid for the impact from Bitcoin to S\&P 500 and Dow Jones. The variation of impulse response from lag 3 to lag 4 has a narrow confidence interval for both S\&P 500 and Dow Jones, which represents high accuracy. Therefore, some absolute correspondence possibly exists between Bitcoin and the stock market.

Table 1 records the results of the variance decompositions for the four variables. In this table, the original change of BT is completely caused by its contribution and its contribution decreases gradually as lag order increases. The growth of contributions of SP, ND, and DJ to BT starts at 
Figure 3. Results of impulse response. From the narrow confidence interval, the strong influences of S\&P 500 and Dow Jones can be seen.

\section{Results of Impulse responses for BT, SP, ND, and DJ}
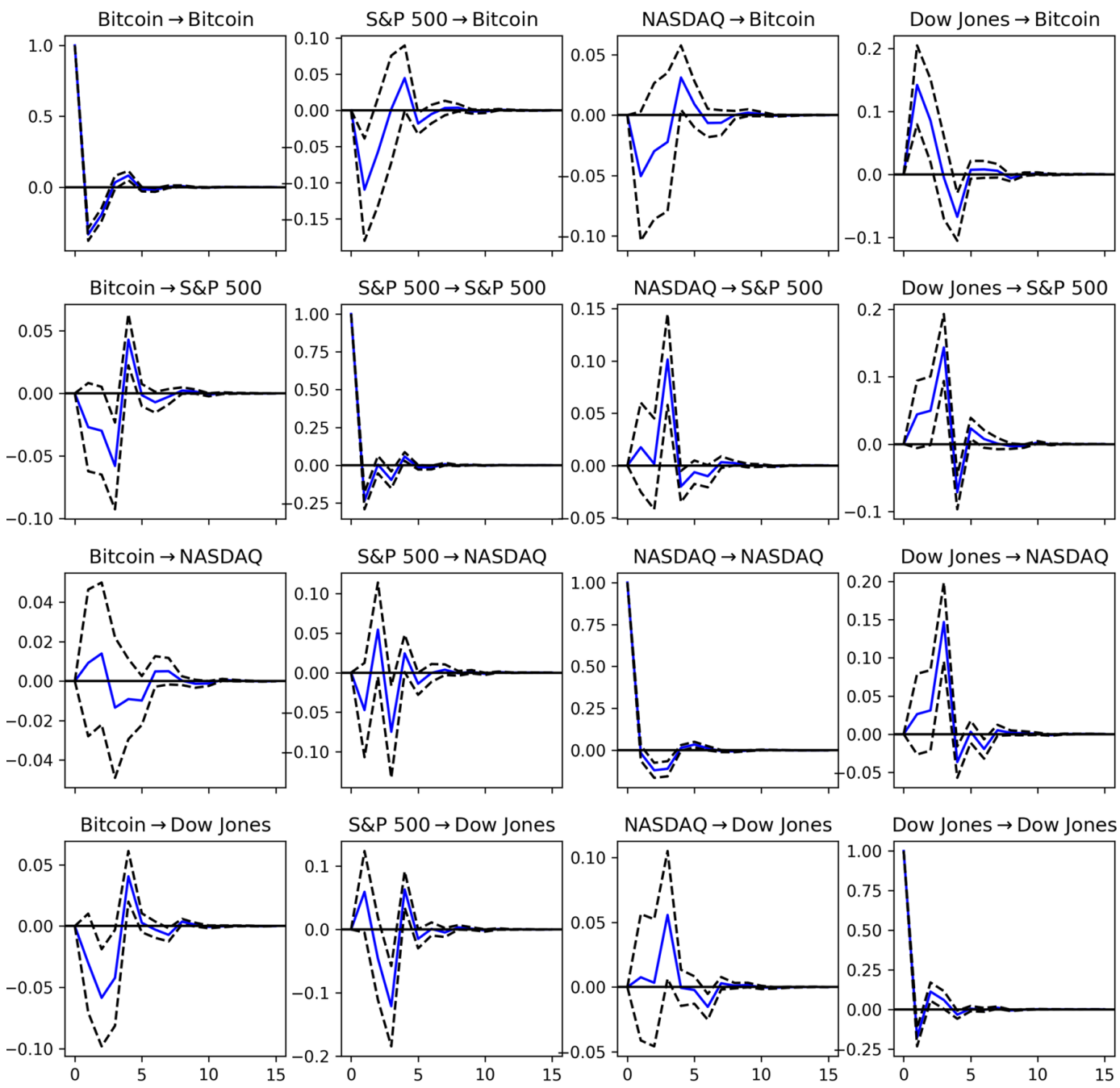

horizon 2 . In the end, the variations are stabilized at $0.0329 \%, 0.1902 \%$, and $1.3667 \%$, respectively, which means that DJ has a comparatively apparent and lasting influence to BT, even though all the effects are not strong enough.

For the change of SP, the contribution of BT, in the beginning, is $0.0023 \%$. The contribution grows up gradually and then stabilizes at $0.8658 \%$. The contributions of ND and DJ start to rise after horizon 2 and stabilize at $1.3805 \%$ and $2.1870 \%$, respectively. It is illustrated that the increment of SP is more likely influenced by the increment of DJ instead of that of BT. No large difference, however, is among the effects of BT, ND, and DJ. For the change of ND, the contribution of BT initially is $0.3888 \%$. It increases gradually and stabilizes at $0.5013 \%$. The contributions of SP and DJ rise gradually and stabilize at 5.0408\% after horizon 10 and $1.6636 \%$ after horizon 12, respectively. This suggests that the increment of DJ has a relatively higher possibility to influence the increment of ND, while all the effects are weak. For the change of DJ, the contribution of BT originally is $0.4796 \%$. It rises gradually and then stabilizes at $1.3409 \%$. The contributions of SP and ND grow up gradually 
Table 1. Variance Decomposition Results. Variance Decomposition results show us the strong influence contribution of S\&P 500 and the opposite shock from S\&P 500 and Dow Jones.

\begin{tabular}{|c|c|c|c|c|c|}
\hline & Horizon & BT & SP & ND & DJ \\
\hline \multirow[t]{6}{*}{ BT } & 1 & 1 & 0 & 0 & 0 \\
\hline & 2 & 0.989973 & 0.000215 & 0.000884 & 0.008928 \\
\hline & 4 & 0.986564 & 0.000240 & 0.001448 & 0.011748 \\
\hline & 7 & 0.984173 & 0.000310 & 0.001880 & 0.013638 \\
\hline & 17 & 0.984102 & 0.000329 & 0.001902 & 0.013667 \\
\hline & 41 & 0.984102 & 0.000329 & 0.001902 & 0.013667 \\
\hline \multirow[t]{6}{*}{ SP } & 1 & 0.000023 & 0.999977 & 0 & 0 \\
\hline & 2 & 0.001041 & 0.997053 & 0.000459 & 0.001446 \\
\hline & 4 & 0.006364 & 0.962605 & 0.013117 & 0.017914 \\
\hline & 7 & 0.008639 & 0.955715 & 0.013791 & 0.021855 \\
\hline & 17 & 0.008658 & 0.955667 & 0.013805 & 0.021870 \\
\hline & 41 & 0.008658 & 0.955667 & 0.013805 & 0.021870 \\
\hline \multirow[t]{6}{*}{ ND } & 1 & 0.003888 & 0.049461 & 0.946651 & 0 \\
\hline & 2 & 0.004069 & 0.050304 & 0.945153 & 0.000474 \\
\hline & 4 & 0.004593 & 0.050400 & 0.929500 & 0.015507 \\
\hline & 7 & 0.004967 & 0.050380 & 0.928034 & 0.016619 \\
\hline & 17 & 0.005013 & 0.050408 & 0.927942 & 0.016636 \\
\hline & 41 & 0.005013 & 0.050408 & 0.927942 & 0.016636 \\
\hline \multirow[t]{6}{*}{ DJ } & 1 & 0.004796 & 0.395261 & 0.005996 & 0.593947 \\
\hline & 2 & 0.006704 & 0.389249 & 0.005901 & 0.598146 \\
\hline & 4 & 0.011659 & 0.385034 & 0.008770 & 0.594537 \\
\hline & 7 & 0.013348 & 0.384793 & 0.008934 & 0.592926 \\
\hline & 17 & 0.013409 & 0.384736 & 0.008946 & 0.592909 \\
\hline & 41 & 0.013409 & 0.384736 & 0.008946 & 0.592909 \\
\hline
\end{tabular}

and stabilize at $38.4736 \%$ after horizon 12 and $0.8946 \%$ after horizon 10, respectively. It is indicated that the increment of DJ is much more likely to be influenced by the increment of SP since SP has strong effect compared to the effect of the other two.

Generally, from the above analyses, it is proved that the relationship between Bitcoin and stock price certainly exists. The effect of shock from Bitcoin is not significant and calms down quickly, while stock market indexes have a comparatively strong influence on Bitcoin. S\&P 500, NASDAQ and Dow Jones, however, have a different effect on Bitcoin's price fluctuation. First of all, S\&P 500 stock growth has a comparatively significant impact on Bitcoin, while the effect influenced by S\&P 500 stock index is weak. Furthermore, stock market indexes influence Bitcoin afterward and the pattern of shock from S\&P 500 or Dow Jones is in the opposition.

\section{Sliding Window Technique}

Sliding window technique is a technique for controlling the window length between two nodes. Yagoubi et al. (2018) proved that the combination between the sliding window and time series can promote to 
find highly correlated pairs of time series over whole windows. As the length of the sliding window changes, the prediction of the time series model will change accordingly.

To further explore the relationship between Bitcoin and stock market based on the three major stock indexes, we lead in the sliding window technique for further study. Through the sliding window technique, the time series period (February 2010 to February 2018) is separated to equal units according to different scales. Every unit together can construct a new time series sequence, in which we calculate the mean and standard deviation for each unit and then obtain eight variables for this new time series sequence: BT_M (mean of Bitcoin), BT_ST (standard deviation of Bitcoin), SP_M(mean of S\&P 500), SP_ST (standard deviation of S\&P 500), ND_M (mean of NASDAQ), ND_ST (standard deviation of NASDAQ), DJ_M (mean of Dow Jones), DJ_ST (standard deviation of Dow Jones). In our experiment, we tested scale equal to 3, 5, 7, and 9 and finally find out the best performance appeared at a scale equal to 5 .

Figure 4 is the plot of impulse response function results on a sliding window when the scale is optimal. The shock effect from BT_M to itself indicates that it has an initially positive strong fluctuation but a negative effect at lag 2. The shock lasts from lag 3 and until lag 20 it flattens away. The effect from SP_ST to BT_M doesn't affect in the beginning and then gradually has a slight positive effect at lag 2. It is worth mentioning that the variation of impulse response caused by SP_ST to BT_M from lag 2 to lag 8 still has a narrow confidence interval and after lag 8 the confidence interval becomes bigger. The effect from DJ_M to BT_M also has a narrow confidence interval from lag 1 to lag 5 . After lag 5, the confidence interval becomes bigger. All the narrow confidence interval appeared in the impulse response from SP_ST or DJ_M means high accuracy, which can further prove some strong correspondence between Bitcoin and the stock market.

Table 2 reports the variance decomposition results performed by sliding window technique. In this result, it suggests that the original change of BT_M is entirely from its contribution. This contribution decreases gradually as lag order rises. The increasing variety of contributions of BT_ST, DJ_M, DJ_ST, ND_M, ND_ST, SP_M, and SP_ST to BT_M starts at horizon 2. Finally, the variations are stabilized at $5.5944 \%, 3.1089 \%, 2.9184 \%, 6.3956 \%, 0.5976 \%, 7.6920 \%$, and $36.9920 \%$, respectively, which means SP_ST has an obvious advantage and lasting influence on BT_M. For the change of BT_ST, the contribution of BT_M is $37.5081 \%$ in the beginning. The contribution rises up gradually and then stabilizes at $37.0126 \%$. The variation of contributions of DJ_M, DJ_ST, ND_M, ND_ST, SP_M, and SP_ST to SP_ST starts at horizon 2 and stabilize at $0.3363 \%, 0.9247 \%, 4.0973 \%, 0.3190 \%, 1.8231 \%$, and $8.5421 \%$, respectively. This indicates that the increment of BT_ST is more possibly influenced by the increment of SP_ST, even though all the effects are not strong enough.

The contributions of BT_M to DJ_M, DJ_ST, ND_M, ND_ST, SP_M, and SP_ST eventually stabilize at $2.3927 \%, 4.8372 \%, 5.3931 \%, 6.6659 \%, 4.9213 \%$, and $6.5005 \%$, respectively. It suggests that the growth of BT_M has a relatively stronger effect on the growth of ND_ST, although no huge difference is among all the effects of contributions. For the change of BT_ST to other stock indexes, the contributions of BT_ST to DJ_M, DJ_ST, ND_M, ND_ST, SP_M, and SP_ST eventually stabilize at $0.9374 \%, 1.2777 \%, 3.2769 \%, 1.6943 \%, 3.5609 \%$, and $1.2317 \%$, respectively, which means the growth of BT_ST has comparatively stronger effect to the growth of SP_M, even though not remarkable enough.

In sum, through involving sliding window, the impulse response signal has been strengthened and the interactive relationship between Bitcoin and stock market index (S\&P 500, NASDAQ, and Dow Jones Industrial Average) has been found. First of all, the standard deviation of S\&P 500 and the mean of Dow Jones both have strong correspondence with the mean of Bitcoin, reviewed from the narrow confidence interval on their impulse response. Secondly, the growth of the standard deviation of S\&P 500 has an outstandingly significant effect on the mean of Bitcoin, while the influences caused by the mean and standard deviation of other indexes are weak. Moreover, the standard deviation of S\&P 500 has a relatively significant effect on the standard deviation of Bitcoin as well. Besides, the mean of Bitcoin has a significantly strong effect on the standard deviation of NASDAQ and the mean of S\&P 500. 
Figure 4. Results of impulse response by Sliding Window. The impulse response signal has a significant enhancement. The narrower confidence internal once again proves the close relationship between Bitcoin and the stock market.

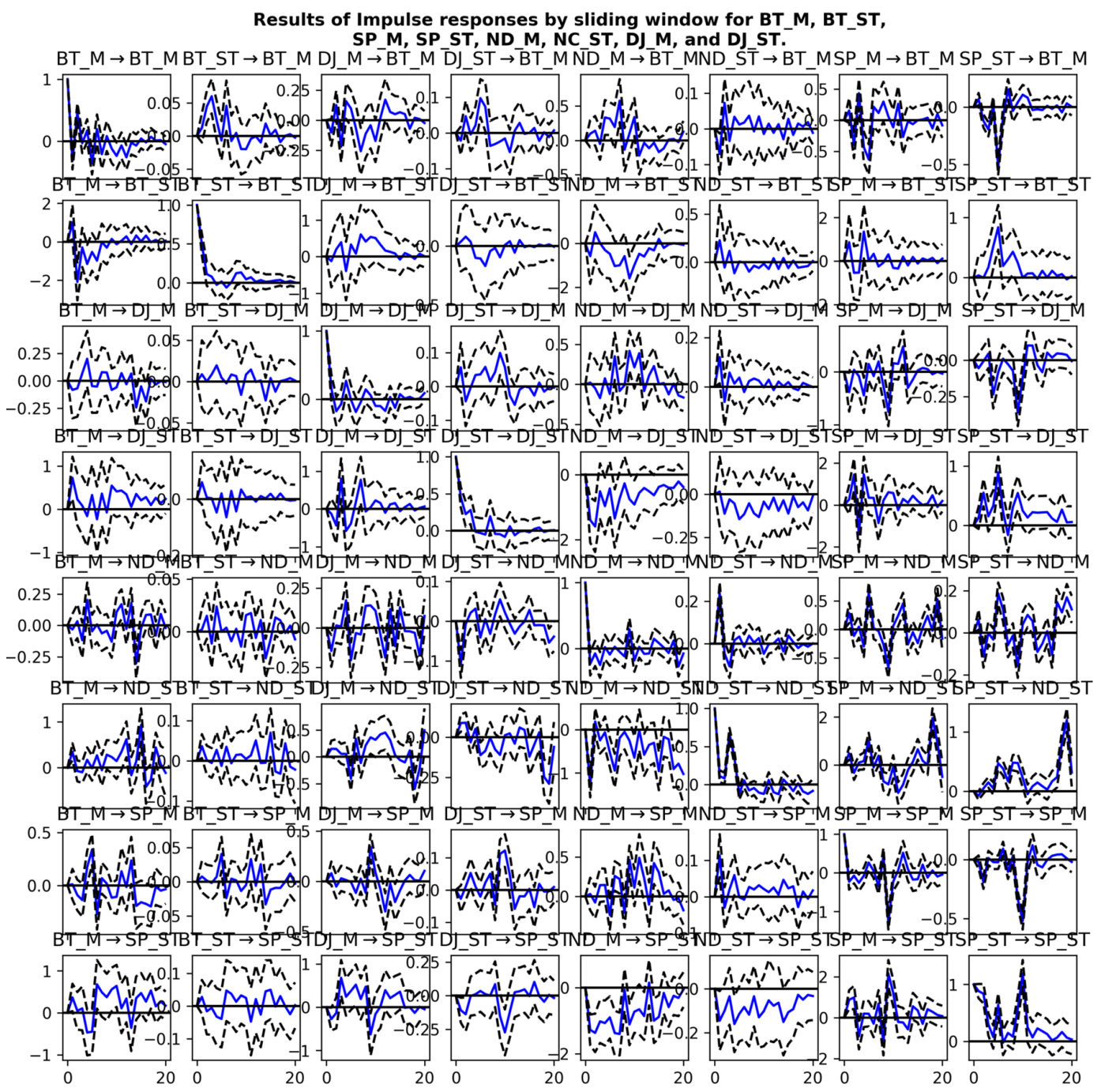

\section{CONCLUSION}

An in-depth exploration and study on the determinate relationship on time series between Bitcoin and the stock market have been conducted by using the VAR model and sliding window technology, which filled up a gap in the financial area. Since Bitcoin and stock market data are both time-series data, the VAR model is useful to forecasting Bitcoin, S\&P 500, NASDAQ, and Don Jones variables. The impulse response results are enhanced through performing sliding window technology, which is a favorable proof of the relationship between Bitcoin and the stock market.

Although the result of impulse response analysis has shown the relationship between Bitcoin and stock market, some vulnerabilities need to be solved to optimize the result in further study. The limitation was caused by sample data itself. Stock data is usually tracked by day, quarter, and second, while Bitcoin data was never recorded in a unit of second. As the result, we couldn't implement wavelet analysis on these two data sources simultaneously in a more detailed time unit. 
Table 2. Variance Decomposition Results by Sliding Window. The results indicate that the growth of the mean of Bitcoin has a strong effect on that of the standard deviation of NASDAQ and the standard deviation of Bitcoin has a strong effect on the mean of S\&P 500 as well.

\begin{tabular}{|c|c|c|c|c|c|c|c|c|c|}
\hline & Horizon & BT_M & BT_ST & DJ_M & DJ_ST & ND_M & ND_ST & SP_M & SP_ST \\
\hline \multirow{7}{*}{ BT_M } & 1 & 1 & 0 & 0 & 0 & 0 & 0 & 0 & 0 \\
\hline & 2 & 0.962996 & 0.003269 & 0.000002 & 0.000598 & 0.011454 & 0.003979 & 0.000983 & 0.016719 \\
\hline & 11 & 0.375929 & 0.054607 & 0.022330 & 0.025657 & 0.058407 & 0.004330 & 0.075286 & 0.383455 \\
\hline & 21 & 0.371889 & 0.055705 & 0.030091 & 0.028790 & 0.063724 & 0.005940 & 0.076393 & 0.367467 \\
\hline & 45 & 0.367291 & 0.055959 & 0.031022 & 0.029163 & 0.063917 & 0.005961 & 0.076881 & 0.369806 \\
\hline & 95 & 0.367010 & 0.055944 & 0.031089 & 0.029184 & 0.063956 & 0.005976 & 0.076920 & 0.369920 \\
\hline & 109 & 0.367010 & 0.055944 & 0.031089 & 0.029184 & 0.063956 & 0.005976 & 0.076920 & 0.369920 \\
\hline \multirow{7}{*}{ BT_ST } & 1 & 0.375081 & 0.624919 & 0 & 0 & 0 & 0 & 0 & 0 \\
\hline & 2 & 0.339666 & 0.649253 & 0.000001 & 0.003617 & 0.000866 & 0.002475 & 0.004006 & 0.000117 \\
\hline & 11 & 0.373838 & 0.476200 & 0.002327 & 0.008490 & 0.036798 & 0.002422 & 0.017247 & 0.082677 \\
\hline & 21 & 0.371162 & 0.471220 & 0.003140 & 0.009015 & 0.040775 & 0.003098 & 0.018006 & 0.083583 \\
\hline & 45 & 0.370184 & 0.469578 & 0.003344 & 0.009241 & 0.040952 & 0.003186 & 0.018219 & 0.085296 \\
\hline & 95 & 0.370126 & 0.469450 & 0.003363 & 0.009247 & 0.040973 & 0.003190 & 0.018231 & 0.085421 \\
\hline & 109 & 0.370126 & 0.469450 & 0.003363 & 0.009247 & 0.040973 & 0.003190 & 0.018231 & 0.085421 \\
\hline \multirow{7}{*}{ DJ_M } & 1 & 0.000712 & 0.001286 & 0.998002 & 0 & 0 & 0 & 0 & 0 \\
\hline & 2 & 0.003542 & 0.002152 & 0.970214 & 0.005334 & 0.004184 & 0.003313 & 0.007642 & 0.003619 \\
\hline & 11 & 0.011852 & 0.005680 & 0.727934 & 0.025789 & 0.025067 & 0.005574 & 0.026214 & 0.171890 \\
\hline & 21 & 0.021598 & 0.008778 & 0.677681 & 0.028797 & 0.041447 & 0.005795 & 0.033919 & 0.181985 \\
\hline & 45 & 0.023889 & 0.009353 & 0.667336 & 0.029244 & 0.042156 & 0.005919 & 0.034713 & 0.187389 \\
\hline & 95 & 0.023927 & 0.009374 & 0.666956 & 0.029258 & 0.042189 & 0.005921 & 0.034770 & 0.187606 \\
\hline & 109 & 0.023927 & 0.009374 & 0.666956 & 0.029258 & 0.042189 & 0.005921 & 0.034770 & 0.187606 \\
\hline \multirow{7}{*}{ DJ_ST } & 1 & 0.002198 & 0.000401 & 0.139889 & 0.857511 & 0 & 0 & 0 & 0 \\
\hline & 2 & 0.022453 & 0.004314 & 0.229018 & 0.705588 & 0.034759 & 0.000091 & 0.000128 & 0.003649 \\
\hline & 11 & 0.029067 & 0.012305 & 0.213574 & 0.431806 & 0.075951 & 0.002639 & 0.040564 & 0.194092 \\
\hline & 21 & 0.045443 & 0.012285 & 0.206626 & 0.400950 & 0.082996 & 0.003862 & 0.039412 & 0.208426 \\
\hline & 45 & 0.048339 & 0.012752 & 0.204472 & 0.396540 & 0.083027 & 0.004062 & 0.040948 & 0.209860 \\
\hline & 95 & 0.048372 & 0.012777 & 0.204384 & 0.396320 & 0.083049 & 0.004078 & 0.040988 & 0.210033 \\
\hline & 109 & 0.048372 & 0.012777 & 0.204384 & 0.396320 & 0.083049 & 0.004078 & 0.040988 & 0.210033 \\
\hline \multirow{7}{*}{ ND_M } & 1 & 0.003055 & 0.000527 & 0.527898 & 0.000042 & 0.468477 & 0 & 0 & 0 \\
\hline & 2 & 0.009607 & 0.002209 & 0.441075 & 0.017509 & 0.405120 & 0.096347 & 0.012349 & 0.015784 \\
\hline & 11 & 0.024200 & 0.026447 & 0.268331 & 0.054301 & 0.250147 & 0.071753 & 0.089607 & 0.215213 \\
\hline & 21 & 0.047231 & 0.030681 & 0.199927 & 0.062966 & 0.188323 & 0.051132 & 0.113375 & 0.306365 \\
\hline & 45 & 0.053756 & 0.032792 & 0.190500 & 0.062738 & 0.174595 & 0.045080 & 0.104372 & 0.336168 \\
\hline & 95 & 0.053931 & 0.032769 & 0.190115 & 0.062684 & 0.174179 & 0.044939 & 0.104271 & 0.337113 \\
\hline & 109 & 0.053931 & 0.032769 & 0.190115 & 0.062684 & 0.174179 & 0.044939 & 0.104271 & 0.337113 \\
\hline \multirow{7}{*}{ ND_ST } & 1 & 0.005265 & 0.014876 & 0.006343 & 0.141592 & 0.061754 & 0.770170 & 0 & 0 \\
\hline & 2 & 0.022001 & 0.014659 & 0.029163 & 0.147281 & 0.216833 & 0.526726 & 0.035446 & 0.007892 \\
\hline & 11 & 0.026032 & 0.017383 & 0.041608 & 0.080900 & 0.142374 & 0.211095 & 0.129873 & 0.350735 \\
\hline & 21 & 0.050040 & 0.019882 & 0.033995 & 0.049331 & 0.116719 & 0.095116 & 0.108819 & 0.526097 \\
\hline & 45 & 0.066748 & 0.016959 & 0.035920 & 0.040740 & 0.156530 & 0.064372 & 0.085883 & 0.532847 \\
\hline & 95 & 0.066659 & 0.016943 & 0.035873 & 0.040596 & 0.156483 & 0.063955 & 0.085737 & 0.533754 \\
\hline & 109 & 0.066659 & 0.016943 & 0.035873 & 0.040596 & 0.156483 & 0.063955 & 0.085737 & 0.533754 \\
\hline \multirow{7}{*}{ SP_M } & 1 & 0.006010 & 0.015373 & 0.613099 & 0.026716 & 0.066343 & 0.007108 & 0.265351 & 0 \\
\hline & 2 & 0.006216 & 0.016497 & 0.590071 & 0.032366 & 0.069295 & 0.018334 & 0.265678 & 0.001543 \\
\hline & 11 & 0.031828 & 0.019847 & 0.205162 & 0.031035 & 0.032704 & 0.010012 & 0.181949 & 0.487464 \\
\hline & 21 & 0.047055 & 0.033607 & 0.201769 & 0.038354 & 0.081129 & 0.011247 & 0.165280 & 0.421559 \\
\hline & 45 & 0.049210 & 0.035522 & 0.198147 & 0.038553 & 0.080948 & 0.012008 & 0.164683 & 0.420928 \\
\hline & 95 & 0.049213 & 0.035609 & 0.197873 & 0.038532 & 0.080950 & 0.012049 & 0.164792 & 0.420982 \\
\hline & 109 & 0.049213 & 0.035609 & 0.197873 & 0.038532 & 0.080950 & 0.012049 & 0.164792 & 0.420982 \\
\hline \multirow{7}{*}{ SP_ST } & 1 & 0.000311 & 0.000130 & 0.053706 & 0.105708 & 0.002715 & 0.000020 & 0.090084 & 0.747326 \\
\hline & 2 & 0.008821 & 0.001643 & 0.116296 & 0.072619 & 0.037331 & 0.000730 & 0.050553 & 0.712007 \\
\hline & 11 & 0.043800 & 0.008005 & 0.060978 & 0.043676 & 0.119481 & 0.003572 & 0.076583 & 0.643905 \\
\hline & 21 & 0.062861 & 0.011915 & 0.075526 & 0.046135 & 0.134021 & 0.005415 & 0.079148 & 0.584980 \\
\hline & 45 & 0.064990 & 0.012314 & 0.075330 & 0.047346 & 0.131953 & 0.005524 & 0.078977 & 0.583565 \\
\hline & 95 & 0.065005 & 0.012317 & 0.075278 & 0.047324 & 0.131894 & 0.005536 & 0.078994 & 0.583651 \\
\hline & 109 & 0.065005 & 0.012317 & 0.075278 & 0.047324 & 0.131894 & 0.005536 & 0.078994 & 0.583651 \\
\hline
\end{tabular}


In this article, we have pointed out the relationship between the stock market and Bitcoin with advanced methods. A structural vector autoregressive model was specified and established for the four variables: Bitcoin, S\&P 500, NASDAQ, Dow Jones. Based on impulse response analysis, it is summarized that the effect by Bitcoin on the stock market is weaker than that by stock market on Bitcoin, while S\&P 500 stock growth has a comparatively strong influence on Bitcoin. Furthermore, the application of sliding window technology facilitates the promotion of impulse response, which can be observed graphically that the growth of standard deviation of S\&P 500 has an remarkably significant effect on the mean of Bitcoin. The VAR model has guided the direction for financial investment based on stock and Bitcoin fluctuations.

In general, the research is of great importance to commercial applications for Bitcoin and stock market. It provides a favorable model reference of VAR model for investment strategies and guides the direction of investment diversity on the strength of impulse response analysis. Additionally, systematic science of sliding window technology promotes enforceability and accuracy of business modeling. Future research efforts will focus on model analysis in a more specialized time unit. By solving the systematic deficiency of data, a more precise investment strategy in a specific period could be achieved.

\section{REFERENCES}

Adenomon, M. O., \& Oyejola, B. A. (2013). Impact of Agriculture and Industrialization on GDP in Nigeria: Evidence from VAR and SVAR Models. International journal of Analysis and Applications, 1(1), 40-78.

Basak, S., Kar, S., Saha, S., Khaidem, L., \& Dey, S. R. (2019). Predicting the direction of stock market prices using tree-based classifiers. The North American Journal of Economics and Finance, 47, 552-567. doi:10.1016/j. najef.2018.06.013

Bringmann, L. F., Ferrer, E., Hamaker, E. L., Borsboom, D., \& Tuerlinckx, F. (2018). Modeling nonstationary emotion dynamics in dyads using a Time-Varying Vector-Autoregressive Model. Multivariate Behavioral Research, 53(3), 293-314. doi:10.1080/00273171.2018.1439722 PMID:29505311

Deb, S. (2019). VAR model based clustering method for multivariate time series data. Journal of Mathematical Sciences, 237(6), 754-765. doi:10.1007/s10958-019-04201-4

Degras, D., Xu, Z., Zhang, T., \& Wu, W. B. (2011). Testing for parallelism among trends in multiple time series. IEEE Transactions on Signal Processing, 60(3), 1087-1097. doi:10.1109/TSP.2011.2177831

Eyal, I., Gencer, A. E., Sirer, E. G., \& Van Renesse, R. (2016). Bitcoin-ng: A scalable blockchain protocol. Usenix. Retrieved from https://www.usenix.org/system/files/conference/nsdi16/nsdi16-paper-eyal.pdf

Eyal, I., \& Sirer, E. G. (2018). Majority is not enough: Bitcoin mining is vulnerable. Communications of the ACM, 61(7), 95-102. doi:10.1145/3212998

Fama, E. F. (1965). The behavior of stock-market prices. The Journal of Business, 38(1), 34-105. doi: $10.1086 / 294743$

Gandal, N., Hamrick, J. T., Moore, T., \& Oberman, T. (2018). Price manipulation in the Bitcoin ecosystem. Journal of Monetary Economics, 95, 86-96. doi:10.1016/j.jmoneco.2017.12.004

Hafezi, R., Shahrabi, J., \& Hadavandi, E. (2015). A bat-neural network multi-agent system (BNNMAS) for stock price prediction: Case study of DAX stock price. Applied Soft Computing, 29, 196-210. doi:10.1016/j. asoc.2014.12.028

Jensen, M. C. (1978). Some anomalous evidence regarding market efficiency. Journal of Financial Economics, 6(2/3), 95-101. doi:10.1016/0304-405X(78)90025-9

Kazem, A., Sharifi, E., Hussain, F. K., Saberi, M., \& Hussain, O. K. (2013). Support vector regression with chaos-based firefly algorithm for stock market price forecasting. Applied Soft Computing, 13(2), 947-958. doi:10.1016/j.asoc.2012.09.024 
Leonarduzzi, R., Rochette, G., Bouchaud, J. P., \& Mallat, S. (2019, May). Maximum-entropy Scattering Models for Financial Time Series. In ICASSP 2019-2019 IEEE International Conference on Acoustics, Speech and Signal Processing (ICASSP) (pp. 5496-5500). IEEE. doi:10.1109/ICASSP.2019.8683734

Malkiel, B. G. (2003). The efficient market hypothesis and its critics. The Journal of Economic Perspectives, 17(1), 59-82. doi:10.1257/089533003321164958

Malkiel, B. G., \& Fama, E. F. (1970). Efficient capital markets: A review of theory and empirical work. The Journal of Finance, 25(2), 383-417. doi:10.1111/j.1540-6261.1970.tb00518.x

Nakamoto, S. (2008). Bitcoin: A peer-to-peer electronic cash system. Retrieved from https://bitcoin.org/en/ bitcoin-paper

Patel, J., Shah, S., Thakkar, P., \& Kotecha, K. (2015). Predicting stock market index using fusion of machine learning techniques. Expert Systems with Applications, 42(4), 2162-2172. doi:10.1016/j.eswa.2014.10.031

Reusens, P., \& Croux, C. (2018). Linearly transforming variables in the VAR model, how does it change the impulse response. Journal of Econometric Methods, 7(1). doi:10.1515/jem-2015-0015

Sadaei, H. J., Guimarães, F. G., da Silva, C. J., Lee, M. H., \& Eslami, T. (2017). Short-term load forecasting method based on fuzzy time series, seasonality and long memory process. International Journal of Approximate Reasoning, 83, 196-217. doi:10.1016/j.jjar.2017.01.006

Selvin, S., Vinayakumar, R., Gopalakrishnan, E. A., Menon, V. K., \& Soman, K. P. (2017, September). Stock price prediction using LSTM, RNN and CNN-sliding window model. In Proceedings of the 2017 International Conference on Advances in Computing, Communications and Informatics (ICACCI) (pp. 1643-1647). IEEE. doi:10.1109/ICACCI.2017.8126078

Yagoubi, D. E., Akbarinia, R., Kolev, B., Levchenko, O., Masseglia, F., Valduriez, P., \& Shasha, D. (2018). ParCorr: Efficient parallel methods to identify similar time series pairs across sliding windows. Data Mining and Knowledge Discovery, 32(5), 1481-1507. doi:10.1007/s10618-018-0580-z

Zhou, X., Pan, Z., Hu, G., Tang, S., \& Zhao, C. (2018). Stock market prediction on high-frequency data using generative adversarial nets. Mathematical Problems in Engineering.

Xin Wang earned her M.S. in Data Science since 2018 and kept doing research in the IntelligentRabbit Al and Big Data Lab since 2019. She will start her PhD in 2020.

Chen Xi, PhD, is a lecturer in the School of Humanity and Law at Beijing University of Civil Engineering and Architecture. She is also a research assistant in Beijing Research Base for Architectural Culture. Her current research interests include English academic writing, settlement evolution and urbanization in China, the U.S., etc.

Peng Zhao is a data science professional with experience in industry teaching, and research. He has a broad range of practical data science experience in different industries, including finance, mobile device, consumer intelligence, big data technology, insurance, and biomedical industries. He is a leading machine learning expertise in a Big Data and Al company in New Jersey. He also manages a data scientist team providing a variety of data consulting services to individuals, businesses and non-profit organizations. 